\title{
Factors associated with the use of percutaneous coronary intervention in elderly Chinese patients with a first ST elevated acute myocardial infarction
}

\author{
This article was published in the following Dove Press journal: \\ Patient Preference and Adherence \\ 17 February 2014 \\ Number of times this article has been viewed
}

\author{
Shan Zhou' \\ Jie Chen ${ }^{2}$ \\ Ru-Yi Xu' \\ Hai-Yun $\mathrm{Wu}^{2}$ \\ 'Chinese Navy General Hospital, \\ Fucheng, Beijing, People's Republic \\ of China; ${ }^{2}$ Institute of Geriatric \\ Cardiology, Chinese PLA General \\ Hospital, Beijing, People's Republic \\ of China
}

\begin{abstract}
Introduction: Rapid reperfusion therapies (RT), particularly percutaneous coronary intervention (PCI), improve short- and long-term outcomes in patients with ST segment elevation myocardial infarction (STEMI). However, a substantial proportion of patients with STEMI, especially older patients, refuse or do not undergo PCI. Our study aims to identify factors associated with the use of PCI in elderly Chinese patients with their first STEMI.
\end{abstract}

Method: Elderly (aged 65 years of age or over) patients with STEMI were enrolled between March 2010 and August 2013 at two hospitals in Beijing. Patients with previous myocardial infarction and those with contraindications to reperfusion were excluded. A standardized questionnaire including onset time and severity of symptoms, history of angina pectoris, comorbid illnesses, functional status, family income, health insurance, education, patients' trust in treating physicians, and whether patient was acquainted with a cardiologist was used to collect data from patients or their family.

Results: Five hundred and sixty-eight patients were enrolled. PCI was accepted by $432(76 \%)$ and refused by 136 (24\%). Multivariate analysis showed that older age ( $>75$ years; odds ratio [OR], $0.57 ; 95 \%$ confidence interval [CI], 0.23-0.78), self-rated mild symptoms (OR, $0.12 ; 95 \% \mathrm{CI}$, $0.06-0.21)$, lower degree of trust in treating physician ( $<6$ in a 10 point scale; [OR, $0.14 ; 95 \%$ CI, 0.09-0.28]), and not being acquainted with a cardiologist (OR, 0.28; 95\% CI, 0.07-0.42) were associated with refusal of PCI.

Conclusion: PCI was refused by almost one quarter of eligible elderly Chinese patients with a first STEMI. Age, symptom severity, and trust in physician were independent factors associated with the use of PCI in these patients.

Keywords: elderly, acute myocardial infarction, percutaneous coronary intervention

\section{Introduction}

For patients with acute myocardial infarction (AMI), particularly those with ST segment elevation myocardial infarction (STEMI), rapid reperfusion therapies (RT) improve both short- and long-term outcomes. ${ }^{1-3}$ In centers with prompt availability and experienced staff, percutaneous coronary intervention (PCI) is usually the preferred modality for establishing coronary reperfusion. ${ }^{1,4}$ Despite PCI's proven benefits and universal guideline recommendations, both previously reported data $^{5-7}$ and our own observations show that a substantial proportion of patients with STEMI who are eligible for RT, especially those at older age, refuse or do not undergo PCI. Therefore, a prospective cohort study was performed of elderly patients with a first STEMI to identify factors influencing the use of primary PCI.
Institute of Geriatric Cardiology, Chinese PLA General Hospital, 28 Fuxing Road, Beijing, People's Republic of China, 100853 Email why4630I@I63.com 


\section{Materials and methods}

Elderly (aged 65 years of age or over) patients were enrolled between March 2010 and August 2013 at the Chinese PLA General Hospital and the Chinese Navy General Hospital. Both hospitals are level 1 first rank hospitals (the most selective and high quality hospitals in the Chinese hospital classification $)^{8}$ located in Beijing, People's Republic of China. Both hospitals are equipped with cardiac catheterization facilities, which can be rapidly accessed by patients, and handle a high volume of coronary interventions $(>3,000$ per year); both hospitals also recommend PCI as the first choice of RT in all eligible patients.

Patients were included if they had ischemic symptoms lasting more than 10 minutes, presented to these two hospitals within 48 hours of those symptoms occurring, and met electrocardiographic criteria for STEMI (ie, persistent ST-segment elevation of $\geq 1 \mathrm{~mm}$ in magnitude in $\geq 2$ contiguous leads or a documented new or presumed new left bundle-branch block). Patients with previous AMI and those with contraindications to reperfusion were excluded from the present study; contraindications that were exclusion criteria included the following: 1) documented or selfreported intracranial neoplasm, arteriovenous malformation, or aneurysm; 2) active bleeding on arrival or within 24 hours; 3) known bleeding diathesis; 4) history of hemorrhagic stroke; 5) active peptic ulcer; 6) recent major surgery/trauma; and 7) suspected or documented aortic dissection.

After the diagnosis of STEMI was confirmed, the treating cardiologist of each patient consulted with the patient and their family. The discussion usually lasted 20-30 minutes and included explanations of the nature and prognosis of the disease, and the efficacy, complications, risks, and cost of different RT. Prior to the PCI procedure, an informed consent signed by the patient or their family, which is mandatory in the People's Republic of China, was obtained, usually by a junior member of the interventional cardiology team who would also be participating in the procedure.

The research associates used a standardized 64-item questionnaire to collect data from patients or their families before or within 3 days of hospitalization. The questionnaire was verbally administered by research associates and included age, sex, symptoms' onset time and severity, history of angina pectoris, comorbid illnesses, functional status, family income, health insurance, education, trust in their treating physicians, and whether they had an acquainted (friend or relative) cardiologist.

Severity of symptoms, whether they were chest pain, chest discomfort, dyspnea, fright or panic, other ischemic related symptoms, or a combination of these, was rated by the patient as severe, moderate, or mild. Functional status was measured by using a Chinese version of the SF-12 scores (12-Item Short Form Health Survey), which itself is an abbreviated version of the SF-36 health questionnaire; this approach has been validated in Hong Kong Chinese by using the same scoring system as the standard SF-12. ${ }^{9}$ Health insurance was categorized as mainstream insurance (including the urban employee basic health insurance scheme, the Government Insurance Scheme and Labor Insurance Scheme) and other insurance (including other noncommercial or commercial insurance). ${ }^{8}$ Currently in the People's Republic of China, the percentage of inpatient medical costs being covered by different health insurance schemes varies greatly, ranging from about $40 \%$ coverage by the New Rural Cooperative Medical Care System, to about $80 \%$ by the Urban Employee Basic Health Insurance Scheme, and to almost $100 \%$ by the Government Insurance Scheme. Physician trust was scored by using the General Practice Assessment Survey (GPAS) trust scale, which is a question asking patients to indicate their overall trust in their physician on a scale from 1 (not at all) to 10 (completely). ${ }^{10}$

Informed consent for this study was obtained from all patients, or their family, by oral communication. Institutional review board approval was obtained at both participating hospitals.

Mean \pm standard deviation, median, and percentages were used in the descriptive statistics. Continuous variables were compared using the Wilcoxon rank-sum test (two-sample test) or Kruskal-Wallis test ( $>$ two-sample test), whereas categorical variables were compared using a chi-squared test. In the univariate analysis, the likelihood that patients underwent PCI was defined as the odds ratio (OR) and 95\% confidence interval (CI). Independent predictors of the use of PCI were determined using logistic regression. All variables that were considered to be associated with PCI use were included in the multivariate model. In the final analysis of the present study, by review of the prior publications, comorbid illnesses included only those considered to have significant influence on patients' decision whether or not to accept PCI, including cancer, previous stroke, dementia, chronic obstructive pulmonary disease, chronic renal failure, hepatic cirrhosis, and disabling arthritis. Patients who underwent angiography, but had anatomy unsuitable for PCI, and patients who had spontaneous reperfusion documented in the infarct-related artery during urgent angiography, were included in the PCI group because their initial acceptance of PCI by these patients. Data were entered using Microsoft 
Excel (Microsoft Corporation, Redmond, WA, USA). Statistical analyses were performed by the Chinese High Intellectualized Statistical Software (CHISS; Yuanyitang Science and Technology, Beijing, People's Republic of China). $P$-values of less than 0.05 were regarded as significant.

\section{Results}

A total of 3,487 patients were admitted to the two hospitals during the enrollment for AMI; 2, 143 (61.5\%) were 65 years of age or older. Among the elderly patients, 878 (41\%) were considered to have STEMI by 12-leads electrocardiography and 696 had no documented or self-reported prior AMI. Of the elderly patients with their first STEMI, 27 died and eleven were transferred to other hospitals before a decision on whether or not to undergo PCI was made. Thirty-four patients refused to participate in this study and $56(8.0 \%)$ were excluded due to contraindications to reperfusion. Finally, 568 patients were enrolled (Figure 1).

The sociodemographic and clinical information of the patients is reported in Table 1 . The overall mean age was $73.6 \pm 8.2$ years and $35.4 \%$ were women. PCI was accepted by $432(76 \%)$ patients or their families and refused by 136 (24\%). Of the 136 patients who declined PCI, 16 (11.8\%) received fibrinolytic therapy.

Univariate analysis showed patients at a younger age (65-74 years) were more likely to accept PCI as compared to those aged 75 years or over; sex, marital status, education, family income, health insurance, symptom onset to arrival time, history of angina, and comorbidities were not associated with the use of PCI. In addition to age, we also found that the severity of symptoms as rated by the patients themselves and their trust in the treating physicians were associated with PCI use. Although only about $11 \%$ of the patients or their families reported they were acquainted with a cardiologist, this factor was significantly associated with the decision to undergo PCI (Table 1).

Multivariate regression analysis results of the factors predicting the use of PCI are shown in Table 2, together with ORs and 95\% CI. After adjustment of all other variables listed in Table 1, patient age, symptom severity, trust, and acquaintance with a cardiologist remain independent predictors of the decision to undergo PCI.

\section{Discussion}

In this prospective study performed at two large Chinese urban hospitals, we found that PCI was refused by almost one quarter of the eligible elderly patients with their first STEMI. We also identified that advanced age ( 75 years of age or over), self-rated mild symptoms, and a lower degree of trust in the treating physician were associated with lower acceptance of PCI; conversely, being acquainted with a cardiologist was associated with higher use of this RT.

Both physician and patient factors may influence the use of PCI. In an earlier study, Schulman et al reported that in the United States, physicians' recommendations for cardiac catheterization were influenced by patients' race and sex of the patient. ${ }^{11}$ The present study was conducted at hospitals where cardiologists are encouraged (by hospital and department administrations) and motivated (by financial rewards and professional competency evaluation) to perform

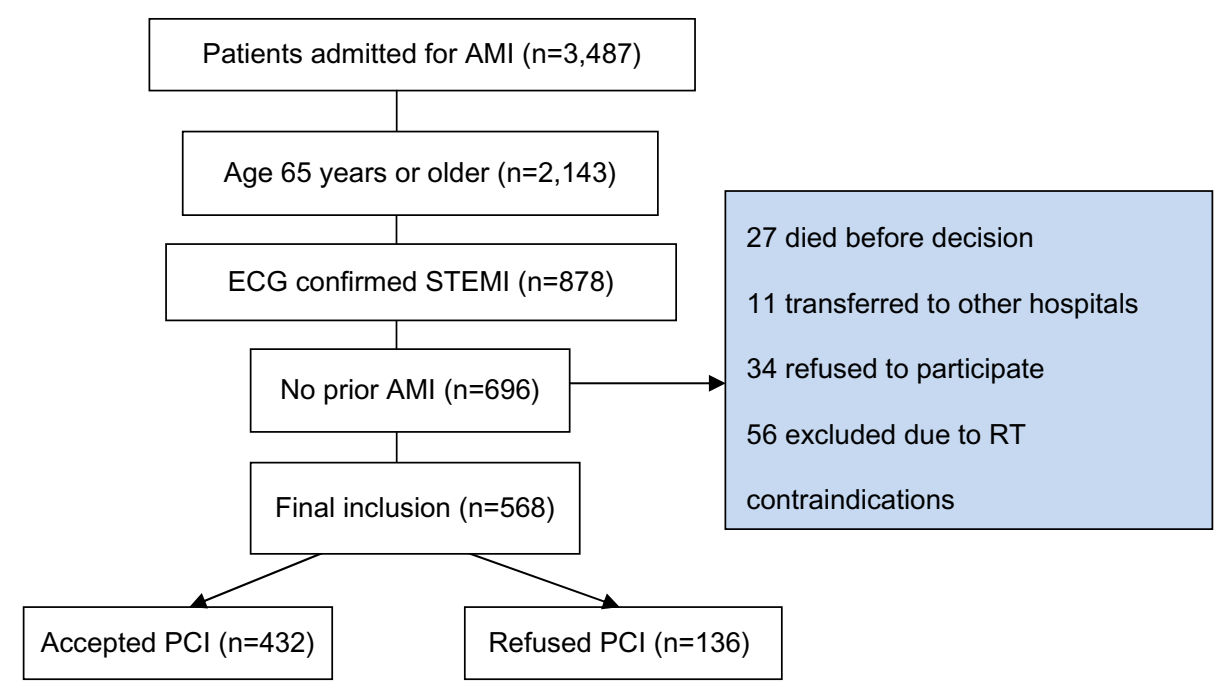

Figure I Selection of study patients.

Abbreviations: AMI, acute myocardial infarction; ECG, electrocardiography; RT, rapid reperfusion therapies; STEMI, ST segment elevation myocardial infarction; PCI, percutaneous coronary intervention. 
Table I Demographic and clinical characteristics of patient by decision to undergo $\mathrm{PCI}$

\begin{tabular}{|c|c|c|c|c|}
\hline & Overall $(n=568)$ & $\mathrm{PCl}(\mathrm{n}=432)$ & No PCl $(n=136)$ & $P$-value \\
\hline \multicolumn{5}{|l|}{ Age, years } \\
\hline $65-74$ & $375(66.0)$ & $304(70.4)$ & 7I (52.2) & \multirow[t]{2}{*}{$<0.01$} \\
\hline$\geq 75$ & $193(34.0)$ & $128(29.6)$ & $65(47.8)$ & \\
\hline \multicolumn{5}{|l|}{ Sex } \\
\hline Male & $367(64.6)$ & $286(66.2)$ & 81 (59.6) & \multirow[t]{2}{*}{0.16} \\
\hline Female & $20 \mathrm{I}(35.4)$ & $146(33.8)$ & $55(40.4)$ & \\
\hline \multicolumn{5}{|l|}{ Marital status } \\
\hline Living alone & $228(40.1)$ & $166(38.4)$ & $62(45.6)$ & \multirow[t]{2}{*}{0.14} \\
\hline Living with spouse/partner & 340 (59.9) & $266(6 \mid .6)$ & $74(54.4)$ & \\
\hline \multicolumn{5}{|l|}{ Education, years } \\
\hline $0-8$ & $416(73.2)$ & $322(74.5)$ & $94(69.2)$ & \multirow[t]{2}{*}{0.21} \\
\hline$\geq 9$ & $152(26.8)$ & $110(25.5)$ & $42(30.8)$ & \\
\hline \multicolumn{5}{|l|}{ Family income (RMB per year) } \\
\hline$<50,000$ & $109(15.2)$ & $88(20.4)$ & $21(15.4)$ & \multirow[t]{3}{*}{0.24} \\
\hline $50,000-100,000$ & $322(56.7)$ & $246(56.9)$ & $76(55.9)$ & \\
\hline$>100,000$ & $137(24.1)$ & $98(22.7)$ & $39(28.7)$ & \\
\hline \multicolumn{5}{|l|}{ Health insurance } \\
\hline Mainstream insurance & $477(84.0)$ & $369(85.4)$ & $108(79.4)$ & \multirow[t]{3}{*}{0.10} \\
\hline Other insurance & $63(11.1)$ & $4 \mid(9.5)$ & $22(16.2)$ & \\
\hline No insurance & $28(4.9)$ & $22(5.1)$ & $6(4.4)$ & \\
\hline Symptom onset to arrival time (minutes, median) & 172 & 168 & 188 & 0.22 \\
\hline \multicolumn{5}{|l|}{ Self-rated severity of symptoms } \\
\hline Mild & $194(34.2)$ & $136(3 \mid .5)$ & $58(42.6)$ & \multirow[t]{3}{*}{$<0.01$} \\
\hline Moderate & $235(4 I .4)$ & $162(37.5)$ & $73(53.7)$ & \\
\hline Severe & $139(24.5)$ & $134(31.0)$ & $5(3.7)$ & \\
\hline \multicolumn{5}{|l|}{ History of angina } \\
\hline Yes & $379(66.7)$ & $287(66.4)$ & $92(67.6)$ & \multirow[t]{2}{*}{0.79} \\
\hline No & $189(33.3)$ & $145(33.6)$ & $44(32.4)$ & \\
\hline \multicolumn{5}{|l|}{ Comorbidities } \\
\hline$\geq 2$ & $184(32.4)$ & $138(3 \mid .9)$ & $46(33.8)$ & \multirow[t]{2}{*}{0.68} \\
\hline$<2$ & $384(67.6)$ & $294(68.1)$ & $90(66.2)$ & \\
\hline \multicolumn{5}{|l|}{ SF-12 score } \\
\hline Lowest quantile & $143(25.2)$ & I0I (23.4) & $42(30.9)$ & \multirow[t]{2}{*}{0.17} \\
\hline Upper 3 quantiles & $425(74.8)$ & $331(76.6)$ & $94(69.1)$ & \\
\hline \multicolumn{5}{|l|}{ GPAS trust scale } \\
\hline$\geq 6$ & $410(72.2)$ & $362(83.8)$ & $48(35.3)$ & \multirow[t]{2}{*}{$<0.01$} \\
\hline$<6$ & $158(27.8)$ & $70(16.2)$ & $88(66.7)$ & \\
\hline \multicolumn{5}{|l|}{ Being acquainted with a cardiologist } \\
\hline Yes & $62(10.9)$ & $56(13.0)$ & $6(4.4)$ & \multirow{2}{*}{$<0.01$} \\
\hline No & $506(89.1)$ & $376(87.0)$ & $130(95.6)$ & \\
\hline
\end{tabular}

Notes: Data are presented as number (\%) except the symptom onset to arrival time; mainstream insurance includes urban employee basic health insurance scheme, Government Insurance Scheme and Labor Insurance Scheme. Percentages may not total 100\% because of rounding.

Abbreviations: PCl, percutaneous coronary intervention; GPAS, General Practice Assessment Survey; RMB, renminbi; SF-12, I2-Item Short Form Health Survey.

Table 2 Regression predictors of decisions against $\mathrm{PCl}$ in elderly (aged 65 years of age or over) patients with first STEMI $(n=568)$

\begin{tabular}{|c|c|c|c|}
\hline & Odds ratio & $95 \% \mathrm{Cl}$ & $P$-value \\
\hline Age $\geq 75$ years & 0.57 & $0.23-0.78$ & 0.03 \\
\hline Self-rated mild symptoms ${ }^{\mathrm{a}}$ & 0.12 & $0.06-0.21$ & $<0.01$ \\
\hline GPAS trust score $<6$ & 0.14 & $0.09-0.28$ & $<0.0$ I \\
\hline No acquainted cardiologist & 0.28 & $0.07-0.42$ & 0.008 \\
\hline
\end{tabular}

Notes: The regression model included all other variables (age, sex, marital status, family income, health insurance, symptom onset to arrival time, education, history of angina, comorbidity, and SF-12 score $)^{\text {a }}$ compared with those with severe and moderate symptoms combined.

Abbreviations: $\mathrm{PCl}$, percutaneous coronary intervention; $\mathrm{Cl}$, confidence interval; GPAS, General Practice Assessment Survey; STEMI, ST segment elevation myocardial infarction. interventional cardiac procedures. Therefore, this study was designed mainly to investigate the influence of patient factors on decision-making.

The underuse of RT in elderly patients with STEMI has been reported in several previous studies. ${ }^{5-7}$ The GRACE Investigators found that patients aged 75 years of age and older were less likely to receive RT (OR: 2.37$)^{5}$ and that coronary angiography and PCI rates decreased significantly with age. ${ }^{6}$ By analyzing data from the CRUSADE initiative, Gharacholou et al found that among eligible STEMI patients, increasing age was the strongest factor 
associated with lack of RT usage (OR of 1.13 per 10 year increase). ${ }^{7}$

We hypothesized that patients with a history of angina pectoris might acquire more knowledge on the treatment of coronary artery disease and thus be more likely to accept PCI, but our analysis did not support this presumption. Traditional Chinese medicine is very popular in the People's Republic of China and remains an important part of the health care system, and "Western medicine" is often criticized by Chinese health professionals as "tending to take a more reductionist approach" and treatment of coronary heart disease has "largely become a catheter and stent craft". ${ }^{12}$ This popular view, although reasonable in some situations, may prevent rather than promote the use of PCI in patients with AMI.

Although several previous researchers suggested that a lack of chest pain might result in patients delaying hospital visits and might be associated with less use of $\mathrm{RT}^{5-7}$ the present study seems to be the first to specifically address the relationship between symptom severity and acceptance of $\mathrm{PCI}$ in patients with AMI. We found patients' self-rated symptom severity was a strong predictor of PCI use in elderly patients with STEMI. This information is new but not surprising patients with more severe symptoms are expected to be more likely to accept aggressive treatments.

In contrast to previously reported results ${ }^{5-7}$ which were performed primarily in Western and industrialized countries, the present study did not find associations between sex, comorbidities, and functional status and the use of PCI. In the People's Republic of China, the average cost of a PCI, including the stent, is about 50,000 RMB (renminbi, or approximately $\$ 7,500 \mathrm{USD}$ ); most patients pay $20 \%-60 \%$ of the cost out of their own pockets. However, we found no association between family income or health insurance type and PCI use. Our subgroup analysis of the present study did not show differences between male and female patients regarding their self-rated symptom severity and trust in their treating physicians, the two strongest factors apart from age (data not shown) influencing decisions. Failing to find a relationship between comorbidities and functional status and PCI use was more difficult to interpret but might be partly explained by the Chinese familial tradition and medical decision-making process. In the People's Republic of China, decisions on major medical intervention in the elderly, particularly those who are frail and severely ill, are often made by sons or daughters, rather than by patients themselves, and "filial piety" asserts a strong influence in decision-making in Chinese societies. ${ }^{13}$ Under this cultural context, family members are unlikely to refuse a treatment for an acute, life-threatening disease when they are convinced that the treatment is beneficial and life-saving, even if the treatment is aggressive or expansive.

We found that patients' and their families' trust in treating physicians was another independent influencing factor of PCI use. Presently, doctor-patient relationships are often described as "in crisis." ${ }^{\prime \prime}$ Although military hospitals are usually viewed by the public as more trustworthy in the People's Republic of China, we found that more than one quarter of the patients and their families scored their trust in physicians 6 or less on a 10-point scale. There is extensive and sometimes false coverage in newspapers, television, and on the Internet that doctors and hospitals conspire to increase charges by providing unnecessary examinations and treatments in the People's Republic of China, particularly through overuse of expensive drugs and devices. A deterioration in the doctorpatient relationship may compromise patients' adherence to physicians' recommendations, even those based on patients' interest.

One interesting finding of the present study was that patients who were acquainted with a cardiologist were significantly more likely to use PCI. In spite of the rise in Western influence during the past century and rapid economic development during the last few decades, the People's Republic of China remains somewhat of an "acquaintance" society and social networks still play an important role. ${ }^{15}$ When facing a catastrophic disease like AMI, patients might seek and accept advice from the cardiologists they are acquainted with, who will in turn usually recommend PCI.

This study has several limitations. First, the study was performed at two large and prestigious military hospitals located in the largest city in the People's Republic of China. It would be reasonable to presume that the average rate of PCI use in elderly patients with AMI would be even lower in the rest of the People's Republic of China. Second, as in many other studies requiring considerations of complex and social factors, we were not able to explore the full reasons behind patient refusal of PCI. For example, in administering the questionnaire, we found that many patients and their families believed that PCI (usually described as "stenting") would do more harm than good, but we did not include this in our study.

\section{Conclusion}

In conclusion, our study confirmed the underuse of RT in elderly patients with STEMI, especially in those with milder symptoms. Lack of trust in treating physicians may be an important driving force in the refusal by patients (or their 
families) of this effective and often lifesaving treatment. Patient education should be promoted, while the communication skills of physicians should be improved to optimize the use of guideline-recommended treatments in patients with AMI.

\section{Disclosure}

The authors report no conflicts of interest in this work.

\section{References}

1. Kushner FG, Hand M, Smith SC Jr, et al. 2009 focused updates: ACC/ AHA guidelines for the management of patients with ST-elevation myocardial infarction (updating the 2004 guideline and 2007 focused update) and ACC/AHA/SCAI guidelines on percutaneous coronary intervention (updating the 2005 guideline and 2007 focused update): a report of the American College of Cardiology Foundation/American Heart Association Task Force on Practice Guidelines. J Am Coll Cardiol. 2009;54(23):2205-2241.

2. Forman D, Wenger NK. What do the recent American Heart Association/ American College of Cardiology Foundation Clinical Practice Guidelines tell us about the evolving management of coronary heart disease in older adults? J Geriatr Cardiol. 2013;10(2):123-128.

3. Franken M, Nussbacher A, Liberman A, Wajngarten M. ST Elevation Myocardial Infarction in the elderly. J Geriatr Cardiol. 2012;9(2): 108-114.

4. Huynh T, Perron S, O’Loughlin J, et al. Comparison of primary percutaneous coronary intervention and fibrinolytic therapy in ST-segmentelevation myocardial infarction: Bayesian hierarchical meta-analyses of randomized controlled trials and observational studies. Circulation. 2009;119(24):3101-3109.

5. Eagle KA, Goodman SG, Avezum A, Budaj A, Sullivan CM, López-Sendón J; GRACE Investigators. Practice variation and missed opportunities for reperfusion in ST-segment-elevation myocardial infarction: findings from the Global Registry of Acute Coronary Events (GRACE). Lancet. 2002;359(9304):373-377.
6. Avezum A, Makdisse M, Spencer F, et al; GRACE Investigators. Impact of age on management and outcome of acute coronary syndrome: observations from the Global Registry of Acute Coronary Events (GRACE). Am Heart J. 2005;149(1):67-73.

7. Gharacholou SM, Alexander KP, Chen AY, et al. Implications and reasons for the lack of use of reperfusion therapy in patients with ST-segment elevation myocardial infarction: findings from the CRUSADE initiative. Am Heart J. 2010;159(5):757-763.

8. Wu HY, Yang LL, Zhou S. Impact of periodic health examination on surgical treatment for uterine fibroids in Beijing: a case-control study. BMC Health Serv Res. 2010;10:329.

9. Lam CL, Tse EY, Gandek B. Is the standard SF-12 health survey valid and equivalent for a Chinese population? Qual Life Res. 2005;14(2):539-547.

10. National Primary Care Research and Development Centre. General Practice Assessment Survey (GPAS) Manual Version 1. Manchester: University of Manchester; 1999.

11. Schulman KA, Berlin JA, Harless W, et al. The effect of race and sex on physicians' recommendations for cardiac catheterization. $N$ Engl J Med. 1999;340(8):618-626.

12. Li LM, Tang JL, Lv J, Jiang Y, Griffiths SM. The need for integration in health sciences sets the future direction for public health education. Public Health. 2011;125(1):20-24.

13. Ho ZJ, Radha Krishna LK, Yee CP. Chinese familial tradition and Western influence: a case study in Singapore on decision making at the end of life. J Pain Symptom Manage. 2010;40(6):932-937.

14. [No authors listed]. Doctors and pharma in China. Lancet. 2013;382(9887): 102.

15. Gold T, Guthrie D, Wank D, editors. Social Connections in China: Institutions, Culture, and the Changing Nature of Guanxi (Structural Analysis in the Social Sciences). New York: Cambridge University Press; 2002.
Patient Preference and Adherence

\section{Publish your work in this journal}

Patient Preference and Adherence is an international, peer-reviewed, open access journal focusing on the growing importance of patient preference and adherence throughout the therapeutic continuum. Patient satisfaction, acceptability, quality of life, compliance, persistence and their role in developing new therapeutic modalities and compounds to

\section{Dovepress}

optimize clinical outcomes for existing disease states are major areas of interest. This journal has been accepted for indexing on PubMed Central. The manuscript management system is completely online and includes a very quick and fair peer-review system. Visit http://www.dovepress.com/ testimonials.php to read real quotes from published authors. 